\title{
The national TB strategy: jointly taking responsibility for TB control?
}

\author{
Onn Min Kon, ${ }^{1}$ Lawrence Peter Ormerod ${ }^{2}$
}

\section{A NATIONAL TUBERCULOSIS STRATEGY}

The 'Collaborative Tuberculosis Strategy for England 2015-2020,1 was recently published on the 19 January 2015 and represents a joint approach by Public Health England (PHE) and the National Health Service of England (NHSE) to tackle the ongoing issue of tuberculosis (TB) in the UK.

Although we saw an encouraging small decrease in the numbers of TB cases in $2013,{ }^{2}$ this is in the setting of this disease continuing to be a problem in the UK where we still have one of the highest rates of TB in Western Europe ${ }^{3}$ and where rates of $\mathrm{TB}$ in some high-burden boroughs are comparable to some areas of the Indian subcontinent and South East Asia. Our rates of disease are still almost double that of the preceding two decades. Despite the epidemiological data indicating that the majority of $\mathrm{TB}$ cases are in foreign-born individuals, the UK has not yet implemented a systematic programme of latent TB infection (LTBI) detection and prophylactic treatment. ${ }^{4}$ In addition, there is evidence that ongoing transmission rates are also extremely high in vulnerable groups such as the homeless, those with substance abuse problems and those with a past history of imprisonment. $^{5}$ The Find \& Treat service based in London has been able to provide a targeted approach in this setting, but similar projects are lacking in other highincidence UK cities.

While the majority of cases and highest rates of disease are centred around the well-recognised 'hot-spots' of TB (in conurbations like London, Leicester, Birmingham, Luton, Manchester and Coventry), there is an emerging issue of complex and multi-drug resistant $\mathrm{TB}$ (MDR-TB) in areas of low incidence where the local clinical infrastructure may be less well resourced and coordinated. Drug resistant $\mathrm{TB}$ is on the increase

\footnotetext{
${ }^{1}$ Chest and Allergy Clinic, St Mary's Hospital, Imperial College Healthcare NHS Trust, London, UK; ${ }^{2}$ University of Manchester, Manchester, UK

Correspondence to Professor Onn Min Kon, Chest and Allergy Clinic, St Mary's Hospital, Imperial College Healthcare NHS Trust, Praed Street, London W2 1NY,
} UK; onn.kon@imperial.nhs.uk globally, with the number of cases of MDR-TB in the UK increasing from $1.1 \%$ $(49 / 4504)$ in 2004 to $1.6 \%(86 / 5397$ cases) in $2011 .^{2}$ Although these numbers are small, they have a disproportionate clinical and resource impact on local services and still continue to pose a challenge to services to complete treatment. The completion rates of these cases at 2 years was only $48 \%$ for the 2011 cohort. $^{2}$

Appropriate commissioning and coordination of TB services is patchy across the $\mathrm{UK}$ and has been an issue for many regions of the country. Funding of TB services is fundamental to the success of TB control and, although there are some difficulties with extrapolating the US experience to the UK, the critical components of appropriate funding and leadership remain partly unmet. Indeed, if we are going to learn from our transatlantic neighbours, they very clearly understood the link between 'taking one's eye off' the 'TB ball $^{6}$ and reaping the consequences of not having a persistent approach to $\mathrm{TB}$ control. In this regard, it is a sober reminder to the UK that the numbers of TB cases in the entire USA may soon be below that of the UK if forecasts of current trends are correct. The US experience would indicate that when funding for $\mathrm{TB}$ control is reduced, TB control suffers as a consequence. Conversely, a funded, focussed and constant approach can result in significant reductions in the numbers of TB cases as demonstrated in New York. ${ }^{7}$ Indeed, the main reason for the very different TB trends between the UK and the USA seems to be the US approach to consistently detect and treat LTBI. ${ }^{8}$

The UK strategy has been developed by the National TB Oversight Group (TBOG) following a 3-month public consultation in 2014. The group is a coalition of key stakeholders from PHE, NHSE, the NHS, the British Thoracic Society, TB Alert, local government, the Department of Health, the Association of Directors of Public Health and the National Institute for Health and Care Excellence (NICE).

The strategy aims to achieve a year-on-year decrease in incidence, a reduction in health inequalities, and ultimately the elimination of $\mathrm{TB}$ as a public health problem in England. To achieve these ambitions and deliver significant improvements in TB control, the strategy sets out the improvements that need to be made in $10 \mathrm{key}$ areas and the actions that are required to achieve these improvements. The key areas of action are: improving access to services and ensuring early diagnosis, providing universal access to high quality diagnostics, improving treatment and care services, ensuring comprehensive contact tracing, improving BCG vaccination uptake, reducing drug-resistant TB, tackling TB in underserved populations, systematically implementing new entrant latent TB screening, strengthening surveillance monitoring, and ensuring an appropriate workforce to deliver TB control. ${ }^{1}$

There is therefore much to be welcomed in the launch of this strategy, and the partnership of NHSE and PHE to deliver this is a significant step forward as the participation of both organisations is vital for improving TB control in England. Programme delivery will be coordinated by a number of regional TB control boards, which are central to this strategy; these will provide over-arching support and an accountability structure for TB control supported by a national TB office. Each board will link to locally focused TB networks and will concentrate their efforts on high-incidence areas while at the same time they will liaise, guide and share their work and expertise with lower incidence areas. This will therefore ensure that there is commitment to fund and coordinate services to meet the demands of local patients and to ensure regions have capacity and expertise to meet the needs of all patients including those with complex disease, in the setting of mixed areas of high and low incidence.

As with the USA, the natural progression of control strategies to include the treatment of LTBI in high-risk groups rather than merely tackling active cases, ${ }^{9}$ has already been modelled and studied in the UK. ${ }^{10} 11$ Commendably, the strategy has committed to ensuring that the detection and treatment of latent TB in new entrants is also resourced. New entrant screening has been recommended by NICE since $2006,{ }^{12}$ with revised thresholds for screening in $2011,{ }^{13}$ but often neither funded nor practised.

There are several challenges that will need particular attention after the launch of the strategy. There will need to be clear accountability and representation from the commissioning elements. The commissioning model is still to be developed and hence, if there are areas with fragmented commissioning rather than a collaborative approach, then the aim of 
providing an appropriate and comprehensive level of care for patients, especially in lower incidence areas, may still be in danger, particularly when there is a lack of mandated commissioning and some clinical commissioning groups for lower TB incidence areas may be under financial pressure not to participate. Individuals with TB may have complex and varying interactions with TB and other related services and hence initiatives to address elements such as housing and access for hard-to-serve populations, will need a broad approach with a Find \& Treat element and multiple stakeholders, especially within the voluntary and housing sectors. ${ }^{14}$ The TB control boards will have to refer to local patterns of disease to ensure their workforce is appropriately funded and coordinated in disparate areas of varying incidence and complexity. The newly defined TB control board areas will also have to ensure that when redefining the 'footprint' of the new areas, that good working clinical and non-clinical pathways are not lost during this process.

The strategy currently only applies to England, where $75 \%$ of non-UK born patients live. ${ }^{2}$ Scotland, ${ }^{15}$ Wales ${ }^{16}$ and Northern Ireland ${ }^{17}$ have lower numbers and incidence, but as $50-55 \%$ of their TB patients are also non-UK born, they too could benefit from a robust policy to detect and treat LTBI, and to ensure appropriate commissioning, funding and staffing. The limited number of TB control boards will mean that some may be too big, or too widely spread geographically, and may split up some currently well-functioning networks that may be arbitrarily divided. For these reasons, some flexibility around the proposed geographical boundaries would be sensible.
Overall, the strategy is to be welcomed and supported, but the 'proof of the pudding will be in the eating'. The challenge will be to ensure that the commissioning and contracting of $\mathrm{TB}$ services ensures that the funding and staffing of TB services is sufficient for its systematic implementation. ${ }^{13} 1418$

Contributors Both OMK and LPO devised and wrote this article.

Competing interests OMK and LPO represented the British Thoracic Society on the National TB Oversight Group.

Provenance and peer review Not commissioned; internally peer reviewed.

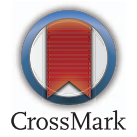

To cite Kon OM, Ormerod LP. Thorax 2015;70:211212.

Received 15 January 2015

Accepted 16 January 2015

Published Online First 3 February 2015

Thorax 2015;70:211-212.

doi:10.1136/thoraxjnl-2015-206808

\section{REFERENCES}

1 Collaborative Tuberculosis Strategy for England 2015-2020. London: Public Health England, Jan 2015. https://www.gov.uk/government/uploads/ system/uploads/attachment_data/file/396263/ CollaborativeTBStrategyEngland_FINAL.pdf.

2 Tuberculosis in the UK: 2014 report. London: Public Health England, Sept 2014.

3 European Centre for Disease Prevention and Control. Tuberculosis surveillance and monitoring in Europe 2013. [Internet]. Stockholm: European Centre for Disease Prevention and Control, 2013. http://www. ecdc.europa.eu/en/publications/_layouts/forms/ Publication_DispForm.aspx?List=4f55ad51-4aed4d32-b960-af70113dbb90\&ID=811

4 Kon OM. Time for a preventative strategy for TB in the UK: further evidence for new entrant screening in primary care. Thorax 2014;69:305-6.
5 Story A, Murad S, Roberts W, et al; London Tuberculosis Nurses Network. Tuberculosis in London: the importance of homelessness, problem drug use and prison. Thorax 2007;62:667-71.

6 Reichman LB. The U-shaped curve of concern. Am Rev Respir Dis 1991;144:741-2.

7 Frieden TR, Fujiwara PI, Washko RM, et al. Tuberculosis in New York City-turning the tide. N Engl J Med 1995;333:229-33.

8 Ormerod LP. Further evidence supporting programmatic screening for, and treatment of latent TB infection (LTBI) in new entrants to the UK from high TB prevalence countries. Thorax 2013;68:201.

9 Macaraig M, Burzynski J, Varma JK. Tuberculosis control in New York City-a changing landscape. N Engl J Med 2014;370:2362-5.

10 Pareek $M$, Watson JP, Ormerod LP, et al. Screening of immigrants in the UK for imported latent tuberculosis: a multicentre cohort study and cost-effectiveness analysis. Lancet Infect Dis 2011;11:435-44.

11 Pareek M, Bond M, Shorey J, et al. Community-based evaluation of immigrant tuberculosis screening using interferon gamma release assays and tuberculin skin testing: observational study and economic analysis. Thorax 2013;69:230-9.

12 National Institute for Health and Clinical Excellence. Clinical diagnosis and management of tuberculosis, and measures for its control and prevention (CG33). London: National Institute for Health and Clinical Excellence, 2006.

13 NICE. Guideline 117. Clinical diagnosis and management of tuberculosis, and measures for its control and prevention (CG117). 2011. http:/ guidance.nice.org.uk/CG117

14 NICE Public Health Guidance. Identifying and managing tuberculosis among hard to reach groups. 2012. http://www.nice.org.uk/ph37

15 Public Health Scotland. Enhanced Surveillance of Mycobacterial IIIness (ESMI) in Scotland 2014. TB annual report for Scotland. http://www.hps.scot.nhs.uk

16 NHS Wales. Enhanced TB Surveillance for Wales: data to end, 2012. http://www.wales.nhs.uk

17 Health Protection Agency Northern Ireland. Epidemiology of tuberculosis in Northern Ireland. Annual surveillance report 2012. http://www. publichealth.hscni.net

18 Tuberculosis case management and cohort review. Guidance for health professionals. London: Royal College of Nursing, Mar 2013. http://www.ren.org. uk/_data/assets/pdf_file/0010/439129/004204.pdf 